\title{
Optical Cavity Effects in ZnO Nanowire Lasers and Waveguides
}

\author{
Justin C. Johnson, ${ }^{\dagger}$ Haoquan Yan,,$+\neq$ Peidong Yang,,,$++\neq$ and Richard J. Saykally $*, \dagger$ \\ Department of Chemistry, University of California, Berkeley, California 94720-1460, and \\ Materials Science Division, Lawrence Berkeley National Laboratory, Berkeley, California
}

Received: February 25, 2003; In Final Form: April 25, 2003

\begin{abstract}
Wide band gap semiconductor nanostructures with near-cylindrical geometry and large dielectric constants exhibit two-dimensional ultraviolet and visible photonic confinement (i.e., waveguiding). Combined with optical gain and suitable resonant feedback, the waveguiding behavior facilitates highly directional lasing at room temperature in controlled-growth nanowires. We have characterized the nanowire emission in detail with high-resolution optical microscopy. The waveguiding behavior of individual zinc oxide ( $\mathrm{ZnO})$ nanowires depends on the wavelength of the emitted light and the directional coupling of the photoluminescence (PL) to the emission dipoles of the nanowire. Polarization studies reveal two distinct regimes of PL characterized by coupling to either guided (bound) or radiation modes of the waveguide, the extent of which depends on wire dimensions. Pumping with high pulse energy engenders the transition from spontaneous to stimulated emission, and analysis of the polarization, line width, and line spacing of the laser radiation facilitates identification of the transverse and longitudinal cavity modes and their gain properties. Interpretation of the lasing spectra as a function of pump fluence, with consideration of $\mathrm{ZnO}$ material properties and ultrafast excitation dynamics, demonstrates a transition from exciton (fluence $<1 \mu \mathrm{J} / \mathrm{cm}^{2}$ ) to electron-hole plasma dynamics (fluence $>1 \mu \mathrm{J} / \mathrm{cm}^{2}$ ) and gain saturation behavior (fluence $>3 \mu \mathrm{J} / \mathrm{cm}^{2}$ ) modified by the constraints of the nanoscale cylindrical cavity.
\end{abstract}

\section{Introduction}

Until recently, the study of experimental nanowire optical physics lagged behind investigations of 0-dimensional and 2-dimensional nanoscale systems (i.e., quantum dots ${ }^{1}$ and boxes/ wells $s^{2,3}$ ). Recent improvements in fabrication techniques for wire and rodlike semiconductor structures have enabled a new generation of experiments on these structures, ${ }^{4-13}$ which are unique because they are capable of exhibiting photonic and/or electronic confinement in two dimensions. Optical and electronic phenomena such as single nanowire logic gates,${ }^{4}$ photodetectors, ${ }^{5,6}$ photochemical sensors, ${ }^{7}$ photonic wire lasers, ${ }^{8}$ and electron-injection lasers ${ }^{9}$ have recently been reported. Initial studies of lasing in nanowire arrays ${ }^{10}$ and single nanowires ${ }^{11,12}$ have provided insight into the phenomenon of confined stimulated emission in these structures, although much of the detailed optical physics remains to be elucidated. It is the goal of the present study to elucidate the nature of the PL waveguiding in individual $\mathrm{ZnO}$ nanowires through the use of microscopy and spectroscopy, and to understand the optical and material aspects of the transition from spontaneous to stimulated emission as it depends on the cavity geometry. The principles guiding the analysis of the optical results are taken from studies on large waveguides (radius $\gg \lambda$ ) and micrometer-sized diode and whispering-gallery mode lasers. ${ }^{15-18}$ A brief summary of the essential points is given below.

Effects due to photonic confinement are observable when the diameter of the nanowire approaches the wavelength of light in the material. The vapor-liquid-solid (VLS) growth mech-

\footnotetext{
* Authors to whom correspondence should be addressed. E-mail: p_yang@uclink.berkeley.edu; and saykally@uclink.berkeley.edu.

University of California, Berkeley.

$\doteqdot$ Lawrence Berkeley National Laboratory.
}

anism $^{14}$ is ideal for producing single-crystal wires that have 40$300 \mathrm{~nm}$ diameters, or $\lambda / 4 n-2 \lambda / n(n=2.45$ for $\mathrm{ZnO})$ for the $\mathrm{UV} /$ blue wavelengths used in this study. On the shorter end of this range, optical confinement becomes reduced due to the influence of diffraction effects. In such cases, coupling of photoluminescence (PL) to axially directed bound waveguide modes is minimal, and the majority of the PL is lost to the surrounding radiation field. This effect can be qualitatively understood by considering the integrated internal and external electromagnetic field intensities for the cylindrical waveguide modes. Roughly, the fractional mode power within the core of the waveguide of radius $r$ is given by ${ }^{15}$

$$
\eta=1-\left(2.405 \exp \left[-\frac{1}{V}\right]\right)^{2} V^{-3}
$$

where $V=k r\left(n^{2}-1\right)^{1 / 2}$ and $k=2 \pi / \lambda$. For wires with $r>100$ $\mathrm{nm},>90 \%$ of the field intensity is retained in the wire for the lowest-order guided mode. However, $<25 \%$ of the field intensity is present inside the wire with $r=50 \mathrm{~nm}$. Consequently, the relative intensities of axially guided versus transversely diffracted light that are detected will differ greatly as a function of the nanowire diameter in this mesoscopic regime. Highly efficient confinement of light within the active medium and minimization of losses to the surroundings are advantageous for the realization of stimulated gain and lasing.

The waveguide modes of the wires can be treated with the aid of classical optical waveguide theory. The quantities of most interest are the transverse components of the electromagnetic fields, which are roughly equivalent to those of a cylindrical optical waveguide. The presence of flat, parallel end faces on the nanowires provides requisite optical feedback for a resonant cavity, analogous to macroscale Fabry-Perot laser resonators. 
The number of bound transverse modes in a multimoded cylindrical optical cavity can be approximated by ${ }^{15}$

$$
M_{\mathrm{bm}}=2(\pi \mathrm{r} / \lambda)^{2}\left(n^{2}-1\right)
$$

in which $r$ is the cross-section radius and $n$ is the refractive index. The value is generally between 1 and 6 for the wires studied here. The cutoff diameter for all higher order transverse modes, or the point at which coupling to radiation versus guided modes is dominant, is about $130 \mathrm{~nm}$ for an ideal $\mathrm{ZnO}$ wire. This cutoff diameter is determined by finding eigenvalues from the wave equation solution, which results in $J_{1}(U)=0$, where $J_{1}$ is a first-order Bessel function and $U$ is a waveguide parameter. The value of $U$ uniquely determines the nanowire diameter at cutoff, given the wavelength and refractive index profile of the waveguide through $U \approx V$, defined above. The number of longitudinal modes per transverse mode that will be observed depends on the width of the gain profile and on the dimensions of the wire. Roughly, the number of longitudinal modes in the Fabry-Perot cavity will be $\Delta v_{\text {spont }} / \Delta v_{\mathrm{c}}$, where $\Delta v_{\mathrm{c}}$ is the spacing of cavity resonances $\left(\Delta \nu_{\mathrm{c}} \approx c / 2 n L\right)$ and $\Delta v_{\text {spont }}$ is the width of the spontaneous emission. This value is $2-10$ for nanowires of typical length $(3-10 \mu \mathrm{m})$. Although these modes may be efficiently guided in the wire, competition for gain may effectively reduce the total number of observed modes to well below the estimated theoretical value.

Each of the transverse modes of the optical cavity has an associated polarization. Much work has been performed on planar microcavities that exhibit waveguiding and lasing, and the modes emitted from these cavities have predominantly transverse electric (TE) or transverse magnetic (TM) character, which is a signature of the quasi-2D geometry. For modes of a cylindrical waveguide, the fundamental $(l=0)$ mode is labeled $\mathrm{HE}_{11}$, which is a hybrid of TE and TM modes. In general, the polarization of the modes is not fixed with respect to the $x$-axis (see Figure 2b), due to the axial symmetry, compared with twodimensional planar lasers. The cross-sections of the $\mathrm{ZnO}$ nanowires, which are actually hexagonal prismatic (see Figure 1), will be treated here as being approximately cylindrical.

Another important issue is the coupling of different types and polarizations of PL to the different cavity modes. Both the position and the orientation of the dipole radiators in the nanowire affect the efficiency of mode coupling. Surface emission couples poorly to guided modes, whereas bulk emission may exhibit either weak or strong coupling, depending on the orientation of the emission dipole with respect to the nanowire symmetry axis. Simulations of nanoscopic dielectric cylinders suggest that radial dipoles couple best to the lowest order $\mathrm{HE}_{11}$ mode and $\mathrm{TE}_{01}$ mode, whereas axial dipoles couple more strongly to the $\mathrm{TM}_{01}$ mode. ${ }^{16}$ Of course, the coupling efficiencies of these modes are also a function of the nanowire dimensions. Due to the crystal symmetry of $\mathrm{ZnO}$, polarized excitation of PL may lead to enhanced coupling of the spontaneous emission to specific nanowire waveguide modes. In this manner, modes can be selectively excited and extracted.

The material physics aspects of the phenomena of $\mathrm{ZnO}$ nanowire lasing are also of great interest both practically and theoretically. Stimulated emission in thin films of $\mathrm{ZnO}$ (band gap $=3.3 \mathrm{eV}$ ) at room temperature was first observed in $1997,{ }^{19}$ although the mechanism of the lasing was somewhat unclear. Random lasing in dense $\mathrm{ZnO}$ particulates has also been proposed and investigated. ${ }^{20}$ Further investigations of $\mathrm{ZnO}$ thin films have revealed strong evidence for the initial onset of lasing being due to exciton-exciton collisions that induce recombination. ${ }^{21,22}$ The large exciton binding energy for $\mathrm{ZnO}$ at room temperature

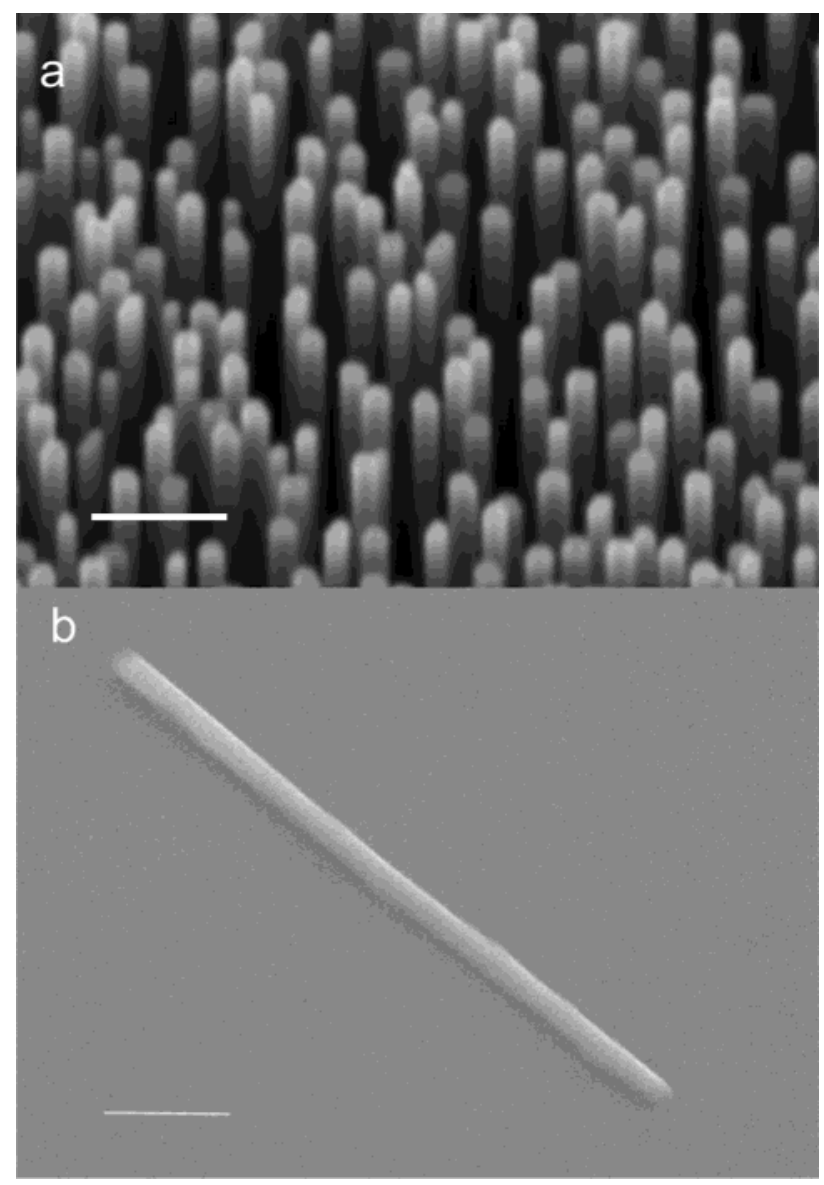

Figure 1. Electron microscopy images of nanowires. SEM image of zinc oxide nanowires (a) as-prepared on a sapphire substrate, and (b) after being dispersed onto a silicon substrate. Scale bar $=1 \mu \mathrm{m}$.

$(60 \mathrm{meV})$ results in excitonic effects prevailing under low excitation conditions. However, as the exciton density increases, the binding energy decreases as excitons are screened due to strong Coulombic interactions with free carriers. When the critical screening length $l_{\mathrm{c}}$ approaches the exciton Bohr radius $a_{\mathrm{b}}$, the exciton becomes destabilized and the material proceeds to an electron-hole plasma (EHP) state. ${ }^{23}$ The existence of exciton versus EHP dynamics in confined structures has been studied recently. ${ }^{24}$ For bulk $\mathrm{ZnO}$, this transition is expected to occur for carrier densities higher than the Mott density

$$
n_{\mathrm{m}} \sim 1.19^{2} k_{\mathrm{b}} T \epsilon \epsilon_{0}\left(e a_{\mathrm{b}}\right)^{-2} \sim 4 \times 10^{18} / \mathrm{cm}^{3}
$$

where $e$ is the charge on an electron. ${ }^{23}$ EHP is thought to be responsible for lasing at room temperature in other materials, such as GaN; however, the gain produced by the EHP recombination is considered to be lower than for exciton-related processes due to the higher efficiency of exciton recombination relative to the bimolecular mechanism of free carrier recombination. ${ }^{25}$ Systems with reduced dimensionality are thought to further stabilize excitons, resulting in the persistence of exciton effects beyond the theoretical Mott density, especially for "quantum" wires. ${ }^{26}$ Although the radii of the wires in our experiments are more than an order of magnitude larger than the exciton Bohr radius for $\mathrm{ZnO}$, the diffusion length of the EHP $(5-10 \mu \mathrm{m})$ is much larger than the typical nanowire diameter. The nanowire cavity could also perturb the carrier mobility, e.g., by forbidding carriers to migrate out of the excitation region, suggesting that some carrier dynamics might be different from those normally encountered in the bulk. 
A shift of the gain curve to lower energies due to band-gap renormalization (BGR) is often observed for increasing pumping intensities, because of an increase in exchange correlations and/ or phase-space filling of the carriers: ${ }^{23} \Delta E_{\mathrm{bg}} \propto-E_{\mathrm{ex}} n_{\mathrm{p}}$, where $\Delta E_{\mathrm{bg}}$ is the shift in band gap energy, $E_{\mathrm{ex}}$ is the exciton binding energy, and $n_{\mathrm{p}}$ is the carrier density. Competing effects due to screening of the exciton binding energy can cause a blue-shift in the gain profile in the excitonic recombination regime, and this can counteract the red-shift due to BGR

$$
\Delta h v=\Delta E_{\mathrm{bg}}+E_{\mathrm{k}}
$$

where $E_{\mathrm{k}}$ is the kinetic energy of the electron-hole pair created. Additionally, cavity resonances can be affected through a change induced in the refractive index of the material. Higher temperatures typically lead to a red -shift of cavity modes because of an increase in refractive index. Typical values of $\mathrm{d} n / \mathrm{d} T$ are approximately $10^{-5} \mathrm{~K}^{-1} \cdot{ }^{27}$ In contrast, saturation of absorption leads to a blue-shift of the modes through a decrease in the real part of the refractive index near the exciton absorption bands. ${ }^{28}$ Here, the cavity resonance frequency $\nu_{\mathrm{c}} \approx \mathrm{cm} /(2 n(\lambda) L)$, where $m$ is the longitudinal mode number, $L$ is the wire length, and $n(\lambda)$ is the refractive index described by a Sellmeier model, $n(\lambda)=A+B \lambda^{2} /\left(\lambda^{2}-C^{2}\right)+D \lambda^{2} /\left(\lambda^{2}-E^{2}\right)$, where the parameters $A-E$ are experimentally determined. ${ }^{29}$ The slope of $n(\lambda)$ is negative in approaching the band edge, thus a blue-shift of the exciton absorption due to saturation causes a decrease in $n$ for a fixed $\lambda$ under high excitation conditions. The carrier dynamics of the highly excited nanowire are of great fundamental interest, ${ }^{30}$ and analysis of the nanolaser spectrum at high pump intensity should lead to new insights into the cavitydependent material behavior.

\section{Experimental Section}

Details of the synthesis of $\mathrm{ZnO}$ nanowires are described elsewhere. ${ }^{31,32}$ Wires were removed from the growth substrate by sonication and dispersed onto several substrates of different refractive index (Al, $\mathrm{Si}$, sapphire, mesoporous silica) by dropcasting a solution of the wires onto the cleaned surface. Scanning electron microscopy (SEM) and transmission electron microscopy (TEM) were used to characterize the structural properties of the wires (Figure 1). Wires prepared for the PL experiments ranged in diameter of $150-500 \mathrm{~nm}$ and had lengths of $5-20 \mu \mathrm{m}$. Wires used in the lasing studies typically had diameters of $100-250 \mathrm{~nm}$ and lengths of $3-10 \mu \mathrm{m}$.

The experimental setup for the nanolaser imaging experiments is diagrammed in Figure 2. PL measurements were made with an unpolarized $\mathrm{He}-\mathrm{Cd}$ laser operating at $325 \mathrm{~nm}$. The pulsed UV radiation $(310 \mathrm{~nm})$ is derived from the frequency-quadrupled output of an optical parametric amplifier (OPA, TOPAS, Quantronix). The OPA is seeded with regeneratively amplified light at $800 \mathrm{~nm}$, from a home-built Ti:sapphire oscillator (88 $\mathrm{MHz})$ and commercial regen/bowtie amplifier $(1 \mathrm{kHz}, 2.5 \mathrm{~mJ}$, Spectra-Physics, Spitfire). The UV pulses are 100-200 fs in duration, with pulse energies of $2-3 \mu \mathrm{J}$. The pulses are attenuated with a variable neutral density filter and the UV is spectrally isolated using a black glass band-pass filter (Edmund, UG11). The beam is directed to either the far-field microscope setup (Figure $2 b$ ) or the near-field scanning optical microscope (NSOM). The geometry for the far-field microscope is depicted in Figure $2 b$. The beam is focused obliquely onto the sample with a $\mathrm{CaF}_{2}$ lens. The spot size is kept relatively large (ca. 200 $\times 100 \mu \mathrm{m})$ to achieve uniform illumination of the nanowires. The light emitted from the sample is collected by a microscope

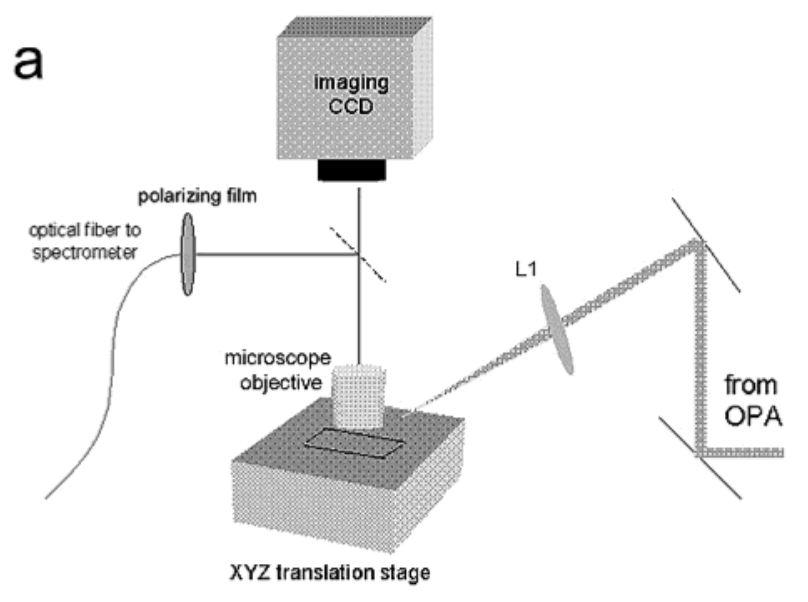

b

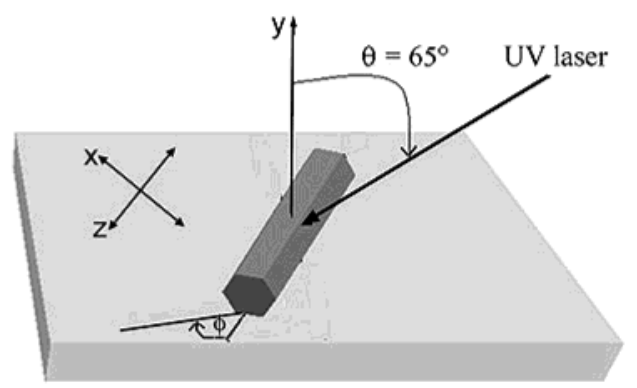

Figure 2. Optical experimental design. (a) Schematic of far-field photoluminescence/lasing experiments. $\mathrm{L} 1=10 \mathrm{~cm}$ focal length $\mathrm{CaF}_{2}$ focusing lens. (b) Magnified view of nanowire orientation. The $z$-direction is defined to be along the major axis of the nanowire, and the $x$-direction is perpendicular to $z$ in the substrate plane. The angle $\phi$ describes the rotation of the nanowire in the $x-z$ plane from the incident plane of the pump laser. $\phi=0$ corresponds to the $z$-axis being perpendicular to the incident plane. The angle $\theta$ describes the azimuthal angle of the pump beam with respect to the surface normal. This angle is fixed at approximately $65^{\circ}$ unless otherwise noted.

objective (Olympus, NA 0.7) and is imaged by a video camera, at a spatial resolution of $0.5-1 \mu \mathrm{m}$. A removable mirror redirects the emission to an optical fiber, which is routed to a spectrograph and CCD (Roper Scientific) for spectral collection. The spectral resolution is about $0.1 \mathrm{~nm}$ for most of the data presented here. A higher resolution system $(0.025 \mathrm{~nm})$ was used for some experiments to explore the lower limit of the nanolaser line width. Polarization measurements were performed by placing either a film polarizer $(3 \mathrm{M})$ or a polarizing cube into the path of the collected signal beam.

The NSOM utilizes a chemically etched single-mode optical fiber for collection of the PL and lasing signals. The details of the design and operation of the NSOM system are described elsewhere. ${ }^{33}$ The near-field spatial resolution is approximately $100 \mathrm{~nm}$, and simultaneous optical and topographic images $(200 \times 200$ pixels $)$ are obtained with each scan.

\section{Results and Discussion}

A. Spontaneous Emission Waveguiding. There are two distinct types of detectable photoluminescence from individual $\mathrm{ZnO}$ nanowires: near-UV free-exciton emission and defect emission. The exciton emission has a bandwidth of approximately $15 \mathrm{~nm}$ and peaks near $385 \mathrm{~nm}$. The defect PL is much broader $($ fwhm $=100 \mathrm{~nm})$ and peaks near $510 \mathrm{~nm}$. The latter emission has been shown to correlate roughly with the 

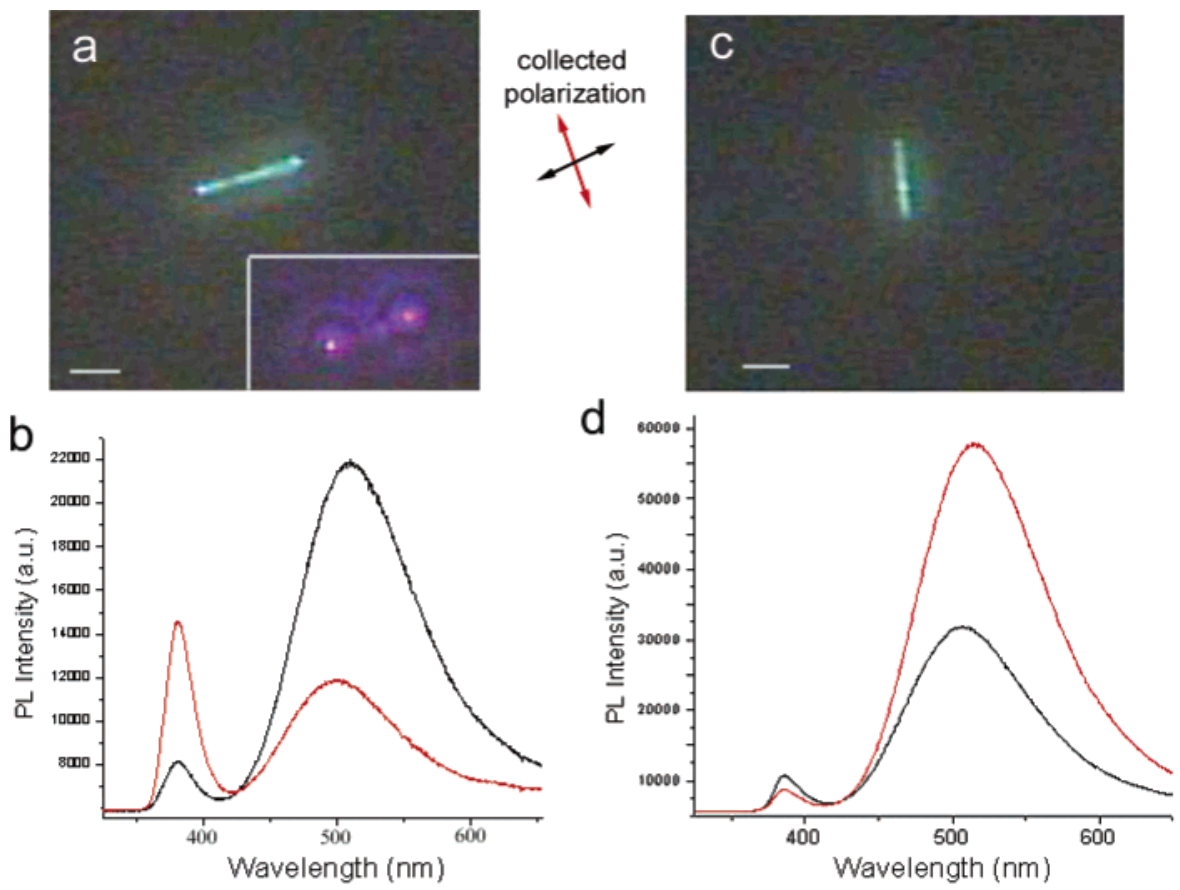

Figure 3. Polarization dependence of spontaneously emitted PL. (a) Far-field image and (b) PL spectrum of an individual ZnO nanowire under unpolarized continuous wave excitation at $325 \mathrm{~nm}(\phi=0)$. Inset: UV-stimulated emission image of the nanowire with pulsed excitation. (c) Far-field image of a nanowire oriented perpendicular $\left(\phi=90^{\circ}\right)$ to the nanowire in (a) and its PL spectrum (d).

concentration of paramagnetic oxygen vacancies in the material $^{34-36}$ (discussed below). Figure 3 shows far-field images of two nanowires oriented at $\phi=0^{\circ}$ and $\phi=90^{\circ}$ and their respective PL spectra, with collection at two orthogonal polarizations, $x$ (radial) and $z$ (axial). For the $x$-polarization in Figure $3 b$, the UV band gap emission is strongly preferred with $\rho_{z}=-0.65$, where $\rho_{z}=\left(\mathrm{I}_{z}-\mathrm{I}_{x}\right) /\left(\mathrm{I}_{z}+\mathrm{I}_{x}\right)$. In contrast, the green emission is preferred for the polarization along the wire major axis $\left(\rho_{z}=0.55\right)$. The same trend is found for the wire in Figure $3 \mathrm{c}$, indicating that the polarization preference is due to the nanowire orientation and not an experimental artifact. Also, PL collected from bulk crystalline $\mathrm{ZnO}$ showed almost no green emission and no polarization preference for the UV emission. The inset of Figure $3 \mathrm{a}$ shows an image of the nanowire illuminated with pulsed excitation, wherein the strong waveguiding of the ultraviolet light to the nanowire ends is evident.

The green PL appears to radiate from all parts of the nanowire and is primarily radially directed, while the UV PL is enhanced near the end of the wire, as shown in Figure $4 d$. Figures $4 a-c$ are near-field images of either the green PL (c) or the UV PL (b) that further illustrate the different behaviors of the exciton and defect emission. Inhomogeneous PL emission along the wire probably indicates a change in the concentration of defects and/ or a change in the scattering efficiency into the NSOM probe.

The apparently anomalous behavior of the polarized emission results from the preference of defect and exciton PL for orthogonal emission dipoles. The waveguiding of the bulk exciton PL suggests that it couples well to guided cavity modes and will be measured as partially polarized in the direction transverse to the nanowire axis ( $x$ and $y$ ) due to the directionality of the emission. However, assuming the PL has equal $x$ - and $y$-polarized components before exiting the nanowire, this emission will be measured as approximately isotropically polarized when analyzed along the $x$ and $z$ directions due to the experimental geometry. The nanowire has uniaxial symmetry with respect to the $z$-axis, so a negative polarization ratio in $x$ and $y$ probably results from polarization-dependent coupling of
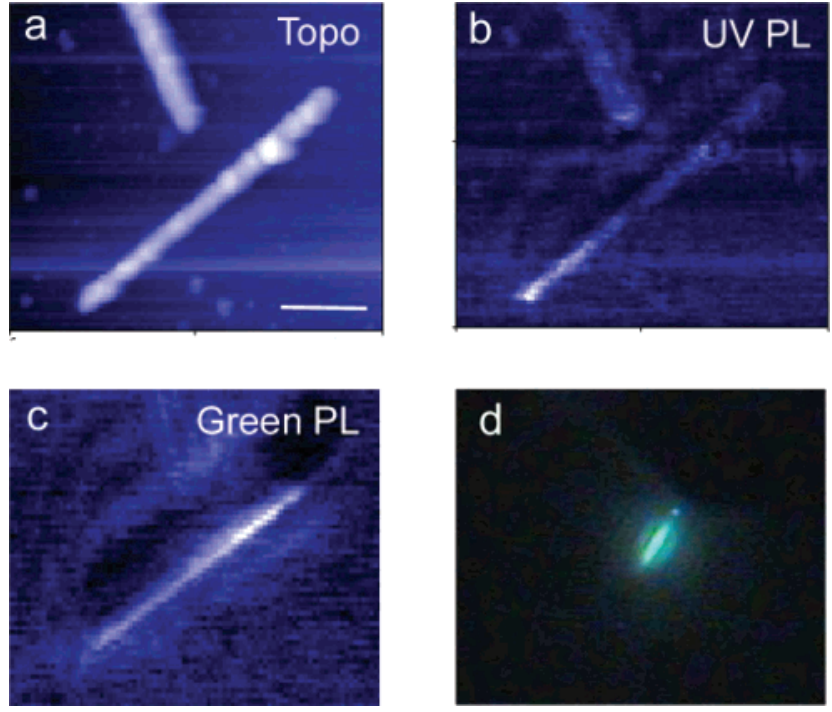

Figure 4. Images of green/UV PL. (a) Topographic, (b) UV, and (c) green near-field PL images. Scale bar $=5 \mu \mathrm{m}$. (d) Far-field image of green/UV PL showing enhancement of UV PL near the wire end. Scale bar $=5 \mu \mathrm{m}$

the waveguided emission to the substrate, as will be discussed in detail in the next section. The efficiency of coupling of the PL to waveguide modes will vary roughly as $1 / \lambda$ for a particular transverse mode, resulting in shorter wavelengths being guided preferentially. Thus, one might expect that the defect PL polarization would be anti-correlated with the degree of mode coupling, given by the fractional mode intensity from eq 1 . Figure $5 \mathrm{~b}$ shows that there is no discernible loss of $\rho$ with increasing diameter, despite the theoretically larger degree of coupling that would exist for the larger diameter wires.

The images of the green defect emission strongly suggest that it is radiated rather than waveguided. In the absence of waveguiding, electromagnetic effects tend to cause the emitted light to prefer a polarization parallel to the long axis of the 

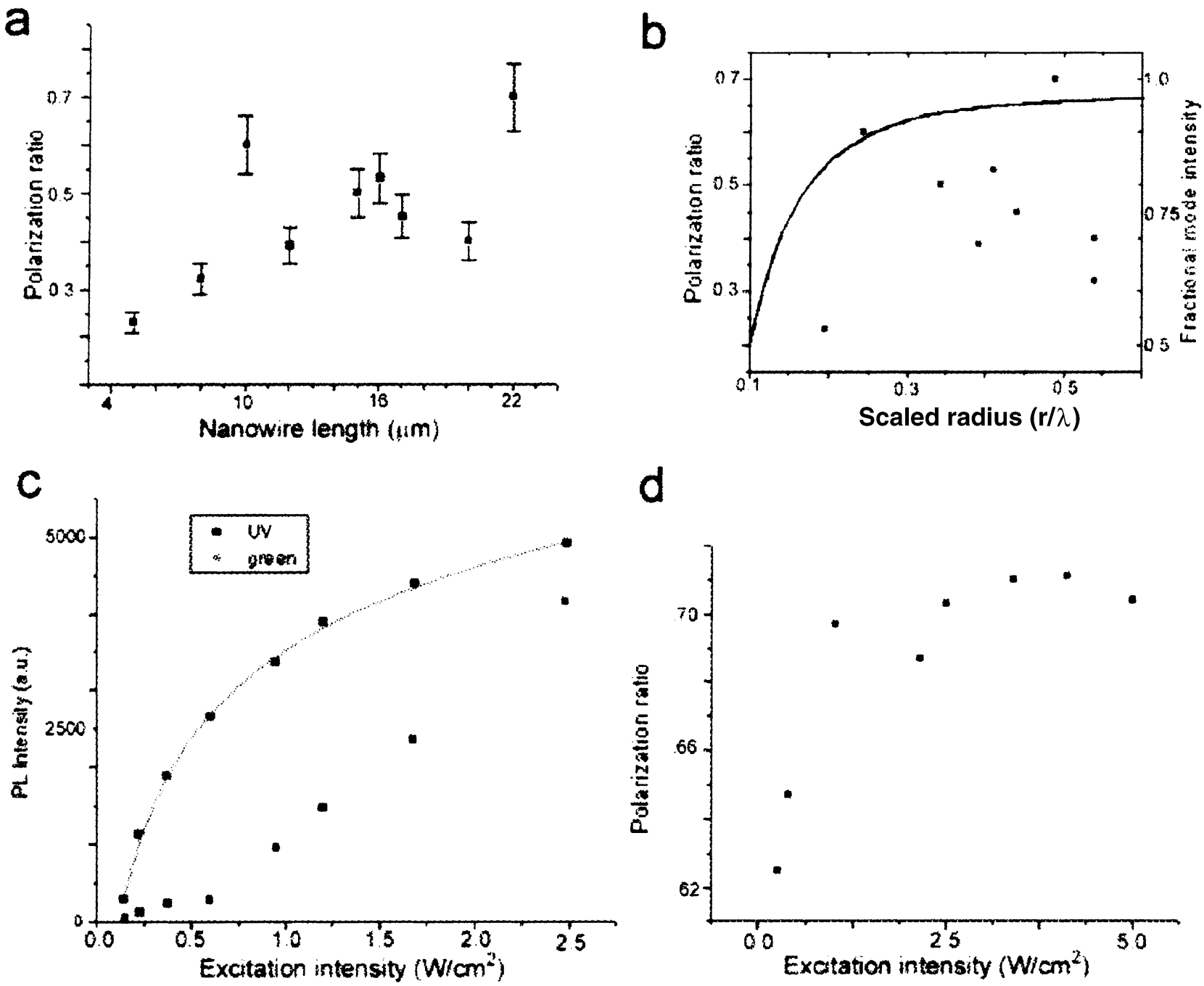

Figure 5. Power dependence of spectrally isolated UV and green PL. (a) Length dependence of polarization ratio for green emission. (b) Diameter dependence of polarization ratio. The solid curve is derived from eq 1, indicating the approximate coupling strength of the defect PL to the lowest order waveguide mode. (c) Overall power dependence of defect PL and UV PL for a single wire. The best-fit curve to the green PL is based on a Langmuir isotherm model. (d) Power dependence of the polarization ratio for a single wire.

wire. ${ }^{37,38}$ In this case, the emission dipole is oriented parallel to the nanowire axis. The magnitude of the reduction of the $x$-polarized field is given by $2 \epsilon_{0} /\left(\epsilon+\epsilon_{0}\right)$, in the limit of an infinitely long and thin wire (where $\epsilon$ and $\epsilon_{0}$ are dielectric constants of $\mathrm{ZnO}$ and vacuum). This would result in a polarization ratio of 0.85 for $\mathrm{ZnO}$. Since the length/diameter ratio for the wires studied is actually about $30-150: 1$, the infinite approximation is not strictly valid, and the predicted polarization ratio is expected to be an upper limit. The highest values of $\rho_{z}$ for the green PL (about 0.75 ) are indeed observed for the longest wires. The plot in Figure 5a exhibits a roughly linear trend of $\rho$ with wire length. The ends of the nanowire are regions that perturb the $z$-polarization, thus longer wires that have a larger percentage of the length that is unperturbed lead to higher values of $\rho$. The precise trend of $\rho$ with length is possibly obscured by variations in the nanowire structure that cause a spread in measured polarization values.

The physical origin of the polarized defect PL preference is not obvious. It is possible that the polarization is due to the approximate surface enhancement of the singly ionized oxygen vacancy, the suggested, though still controversial, origin of the green defect PL in zinc oxide. EPR ${ }^{34}$ results have identified a correlation between the strength of defect emission and the concentration of paramagnetic (singly ionized) oxygen vacancy defect centers $\left(\mathrm{V}_{\mathrm{o}}{ }^{\bullet}\right)$. Transient $\mathrm{PL}^{36}$ results suggest that these $\mathrm{V}_{\mathrm{o}} \cdot$ exist primarily in the thin $(\sim 30 \mathrm{~nm})$ electron-depletion layer near the surface of $\mathrm{ZnO}$ grain boundaries that arises due to bandbending. $\mathrm{V}_{\mathrm{o}}$ is capable of capturing a free electron or combining with an acceptor (such as $\mathrm{V}_{\mathrm{ZN}}{ }^{-}$), and emitting green light according to

$$
E_{\mathrm{DAP}}=E_{\mathrm{g}}-E_{\mathrm{D}}-E_{\mathrm{A}}+e^{2} /(\epsilon d)
$$

where $d$ is the average distance between donor and acceptor, $\epsilon$ is the dielectric constant, and $E_{\mathrm{g}}, E_{\mathrm{A}}, E_{\mathrm{D}}$ are the energies of the band gap, acceptor, and donor, respectively. ${ }^{28}$ The results here show that the defect emission saturates at high excitation intensities (Figure 5b), whereas the UV PL signal shows a nearly linear increase with pump intensity. The power dependence of the green PL fits well to a $I_{\mathrm{PL}} \propto K I_{\mathrm{p}} /\left(1+K I_{\mathrm{p}}\right)$ dependence, where $K$ is the fitted ratio of the trapping rate to loss rate for carriers at a defect site, as in the Langmuir isotherm model. The close agreement indicates that there is a competition between an increase in the number of carriers which can migrate to the defects and a decrease in the number of defects available as sites for recombination as electrons are trapped at those sites.

The other noticeable feature in the polarized spectrum is the shifting of peak wavelength $\lambda_{\max }$ of the green emission. On the 

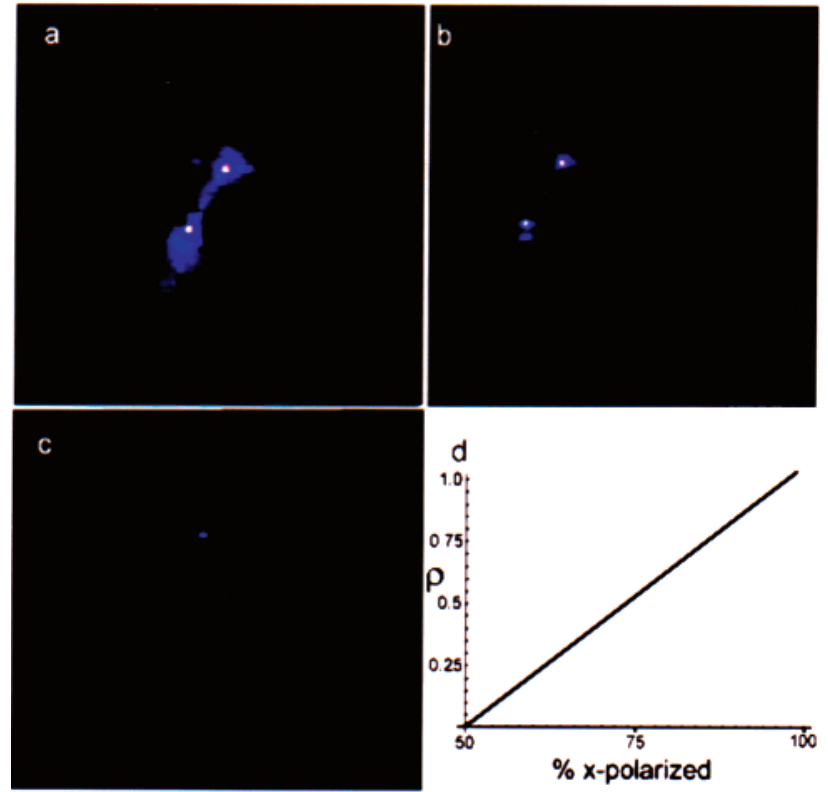

Figure 6. Far-field images of nanowires excited with $200 \mathrm{fs}$ pulses at $310 \mathrm{~nm}$. (a) Far-field image showing laser emission from a single nanowire, all polarization is collected. (b) Far-field image with only $x$-polarized light detected. (c) Far-field image with only $y$-polarized emission detected. (d) Simulation of the detected polarization ratio of the lasing light as a function of the percentage of $x$-polarized PL inside the nanowire cavity.

average, $\lambda_{\max }$ shifts by $10 \mathrm{~nm}(70 \mathrm{meV})$ to shorter wavelength for the $x$-polarized emission. The residual defect PL that does not adopt the z-polarization will be partially waveguided, and due to the wavelength dependence of the guiding efficiency, shorter wavelengths would show a slightly larger value of $\rho$. This is clearly observed, and $\rho$ is $2-3 \%$ larger at $510 \mathrm{~nm}$ than at $500 \mathrm{~nm}$. Another consideration is that shallower traps, such as neutral $\mathrm{V}_{\mathrm{o}}$, are more likely to be activated in the bulk than at the surface, where the Fermi level has been reduced with respect to the conduction band. Thus, higher energy PL resulting from these shallower traps is more bulklike than the PL from deeper traps and might be preferentially waveguided. The inherently broad nature of the defect PL prohibits direct identification of the various defect species; however, the degree of shifting of the PL maximum $(50-90 \mathrm{meV})$ is fairly consistent with the predicted energy level difference between $\mathrm{V}_{\mathrm{o}}$ and $\mathrm{V}_{\mathrm{o}} \cdot 35$

It is also interesting to consider the power dependence of the polarization ratio, since the width of the depletion layer is thought to be a function of the incident power. ${ }^{36}$ The plot of $\rho$ versus incident intensity for a single wire in Figure 5c shows a trend of increasing polarization ratio with pump intensity, and the effect reaches a steady-state near $\rho_{z}=0.71$. The change is only about $10 \%$ over the power range studied, but it is consistently observed in several wires. The reason may be due to a further thinning of the depletion layer with increasing carrier density, causing an even greater surface enhancement of the defect emission. This results in poorer coupling of the green PL to the waveguide modes. This is indicated in the earlier onset of saturation in the $x$ - versus $z$-polarized green PL power dependence (Figure 5c).

B. Stimulated Emission Polarization. Figure 6 shows farfield images of nanowires excited with a femtosecond UV pump. The UV-stimulated emission is clearly dominant over the green $\mathrm{PL}$ in this excitation regime. The emission pattern seen in Figure $6 \mathrm{a}$ is typical for nanowire lasers. Figures $6 \mathrm{~b}$ and $6 \mathrm{c}$ show images taken at two orthogonal polarizations, labeled $x$ and $y$. Integrat- ing the entire lasing spectrum at the two polarizations gives the polarization ratio $\rho_{x}=\left(\mathrm{I}_{x}-\mathrm{I}_{y}\right) /\left(\mathrm{I}_{x}+\mathrm{I}_{y}\right)$ for the nanolaser. Polarization ratios for the wires dispersed on mesoporous silica $(n=1.2)$ were found to be consistently $0.40 \pm 0.05$. For emission directed along the nanowire $z$-axis, the polarization ratio measured should be near zero if the nanolaser light is initially unpolarized. The significant polarization ratio observed here is likely due to a polarization-dependent coupling to the substrate as discussed above. To account for the observed value of $\rho_{x}$, a preference of the stimulated emission inside the cavity for $x$-polarization must be present. The expected $\rho_{x}$ as a function of the degree of $x$-polarization of the emitted nanolaser beam is shown in Figure 6d. The measured values of $\rho_{x}$ should be considered as a lower limit for their values inside the nanowire cavity.

For a nanowire having no coupling to the substrate, the laser modes may be polarized, but they would not exhibit a consistent polarization with respect to a fixed axis, as is observed here due to the asymmetry introduced by the substrate. On sapphire $(n=1.8)$, the polarization ratios were found to vary to a larger degree, with some values reaching 0.6. Although it is difficult to directly compare the results from wires on different substrates since the exact amount of substrate contact varies with each wire, the higher $\rho_{\text {tot }}$ could result from wires with a larger degree of substrate coupling that results in a stronger preference for $x$-polarization (due to Fresnel losses from reflection at the $\mathrm{ZnO} /$ sapphire boundary). As discussed in reference 11, we observe that many nanowires on the sapphire substrate show quenched lasing unless the substrate contact is limited. Mesoporous silica has a lower refractive index, thus it is not surprising that a larger fraction of the wires on this substrate exhibit lasing.

The relative polarizations of the individual cavity modes with respect to each other in some cases identifies the transverse mode with which they are associated. The results in Figure 7 demonstrate this effect. Four modes are observed in the lasing spectrum for the single wire, and the polarization of the modes is analyzed. Alternating modes show similar polarizations, with one pair showing little polarization preference $\left(\rho_{x}<0.03\right)$, and the other pair showing emission polarized parallel to the substrate $\left(\rho_{x}=0.27,0.18\right)$. The polarized modes are likely to be $\mathrm{HE}$ modes, possibly $\mathrm{HE}_{11}$ and/or $\mathrm{HE}_{12}$, the fundamental modes for a cylindrical waveguide. The unpolarized modes are likely to be TE and/or TM modes, the next lowest order modes. These modes have a polarization that changes along the cross section of the nanowire, and these changes would not be resolved in the experiment, resulting in effectively random polarization. The spacing between modes of similar $\rho$ is approximately $2.3 \mathrm{~nm}$, which is in reasonable agreement with expected longitudinal mode spacing ${ }^{39}$ given by

$$
\Delta \lambda=\frac{\lambda^{2}}{2 L\left(n-\lambda \frac{\mathrm{d} n}{\mathrm{~d} \lambda}\right)}
$$

where $L=9 \mu \mathrm{m}, n=2.4$, and $\lambda \mathrm{d} n / \mathrm{d} \lambda$ is approximately $1.0 .^{29}$ The spectra in Figure 7c are taken at higher pump intensity, and the polarization ratios are diminished. Since all modes show the same behavior, it is likely that the decrease in $\rho$ is due to an increase in unresolved $x$-polarized background (from higher order modes or ASE).

Figures $8 \mathrm{a}$ and $8 \mathrm{~b}$ demonstrate the orientation dependence of cavity mode excitation. The spectra in Figures $8 \mathrm{a}$ and $8 \mathrm{~b}$ were collected from the same nanowire, but with the sample 

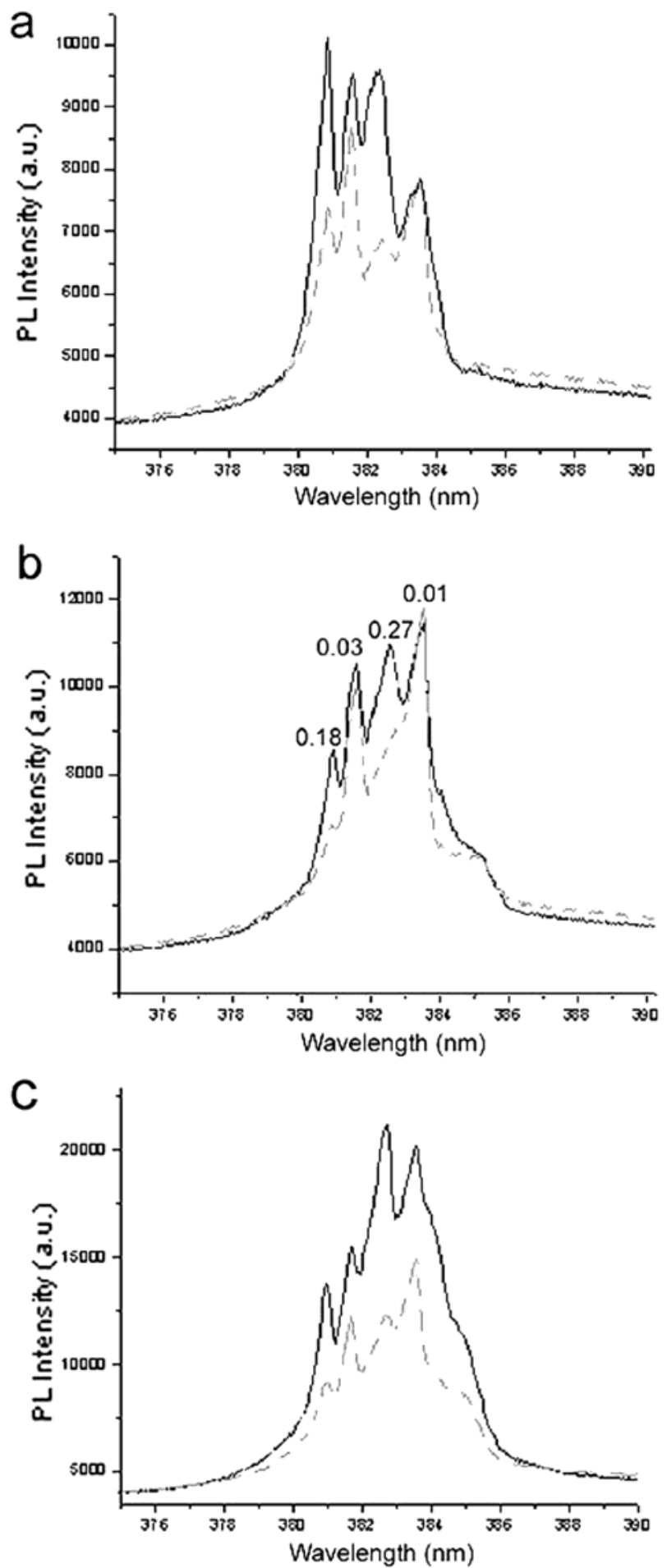

Figure 7. Polarization of individual laser modes for a nanowire with multimode emission $(d \sim 200 \mathrm{~nm})$. (a) - (c) Lasing spectra collected analyzing $x$ (solid)- and $y$ (dashed)-polarized emission at pump fluences of $500 \mathrm{~nJ} / \mathrm{cm}^{2}, 700 \mathrm{~nJ} / \mathrm{cm}^{2}, 1200 \mathrm{~nJ} / \mathrm{cm}^{2}$, respectively. The polarization ratios of each mode are given.

rotated by $90^{\circ}$. The dominant mode shows the highest degree of polarization selectivity in both spectra and is thus labeled $\mathrm{HE}_{11}$ (it also shows the lowest threshold as shown in Figure 8a, inset). New modes appearing in Figure $8 \mathrm{~b}$ appear to be a pair of longitudinal modes from the same transverse mode since their polarization ratios match, and they are spaced at about $5 \mathrm{~nm}$, which is approximately the predicted mode spacing for $L=5 \mu \mathrm{m}$.
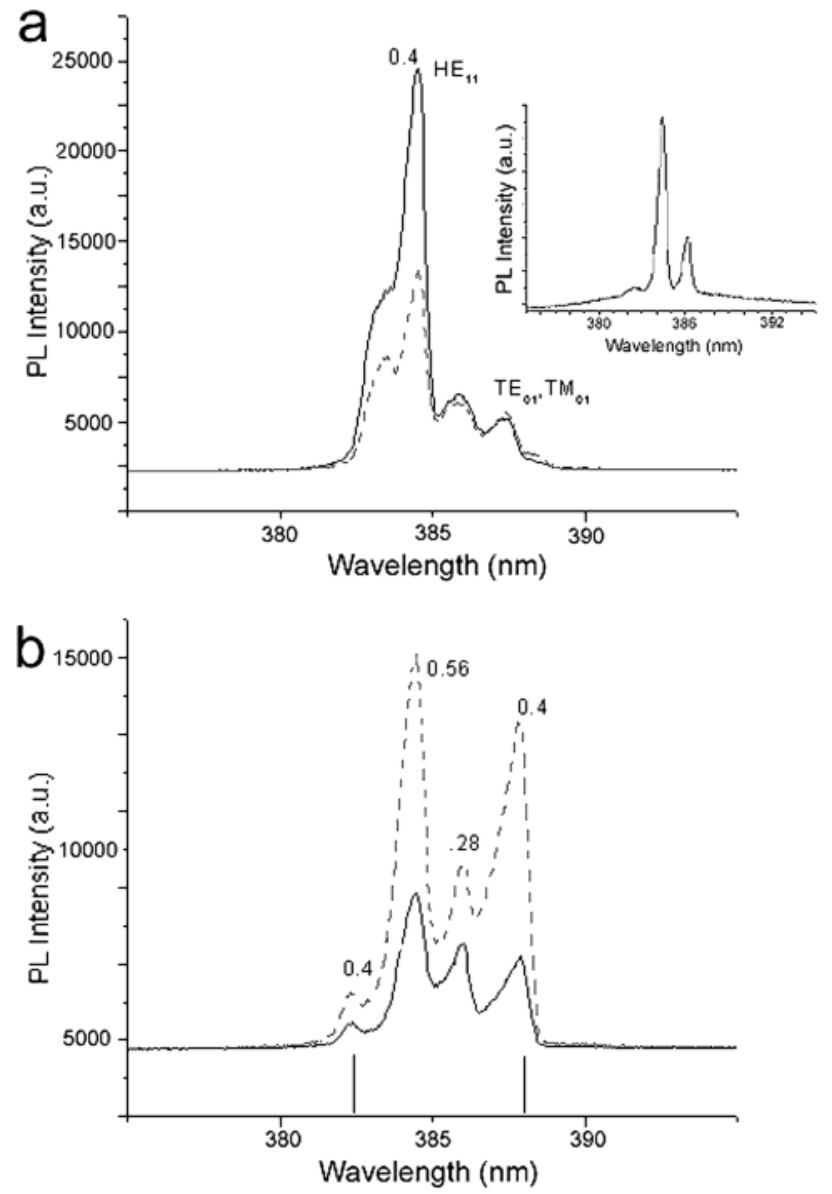

Figure 8. Polarization of individual laser modes as a function of nanowire orientation. (a) Spectra collecting $x$ (solid)- and $y$ (dashed)polarized emission with $\phi=0$. Inset: spectrum near threshold showing the lowest threshold mode. (b) Spectra with nanowire oriented at $\phi=$ $90^{\circ}$. The dashed curves are for the same polarizer position in both (a) and (b). The solid curves are for a polarizer position perpendicular to that corresponding to the dashed curve.

Figure $9 a-c$ demonstrates the effect of selective excitation of different dipoles. Figure 9a shows a spectrum with an incident polarization oriented at $\phi=0$. In this case, HE, TE, and TM modes could be potentially excited. The strongest mode also has the largest value of $\rho$, and is thus labeled $\mathrm{HE}_{11}$. In Figure $9 \mathrm{~b}$, only the axial dipole is excited, which theoretically should couple poorly to the waveguide TE modes. The mode at 380 $\mathrm{nm}$ is observed to disappear while the other modes remain. In Figure 9c only the radial dipole is excited (parallel to the substrate), thus the TE mode again is excited and the TM mode diminishes.

The emission pattern emerging from the lasing nanowire also contains information about the modes excited within the cavity. Diffraction at the wire end face causes the emission to diverge, resulting in a quasi-cone of emission with the approximate half angle 40

$$
\phi=\lambda / \pi r
$$

The lowest order modes have a nearly Gaussian electromagnetic intensity profile within the cavity, which results in the exiting emission peaking along the nanowire axis (see Figure 10). However, higher order transverse modes have electromagnetic field profiles with an angular dependence, leading to angular maxima and minima in the diffracted cone. For example, $\mathrm{TE}_{01}$ and $\mathrm{TM}_{01}$ modes would have a local minimum of intensity 

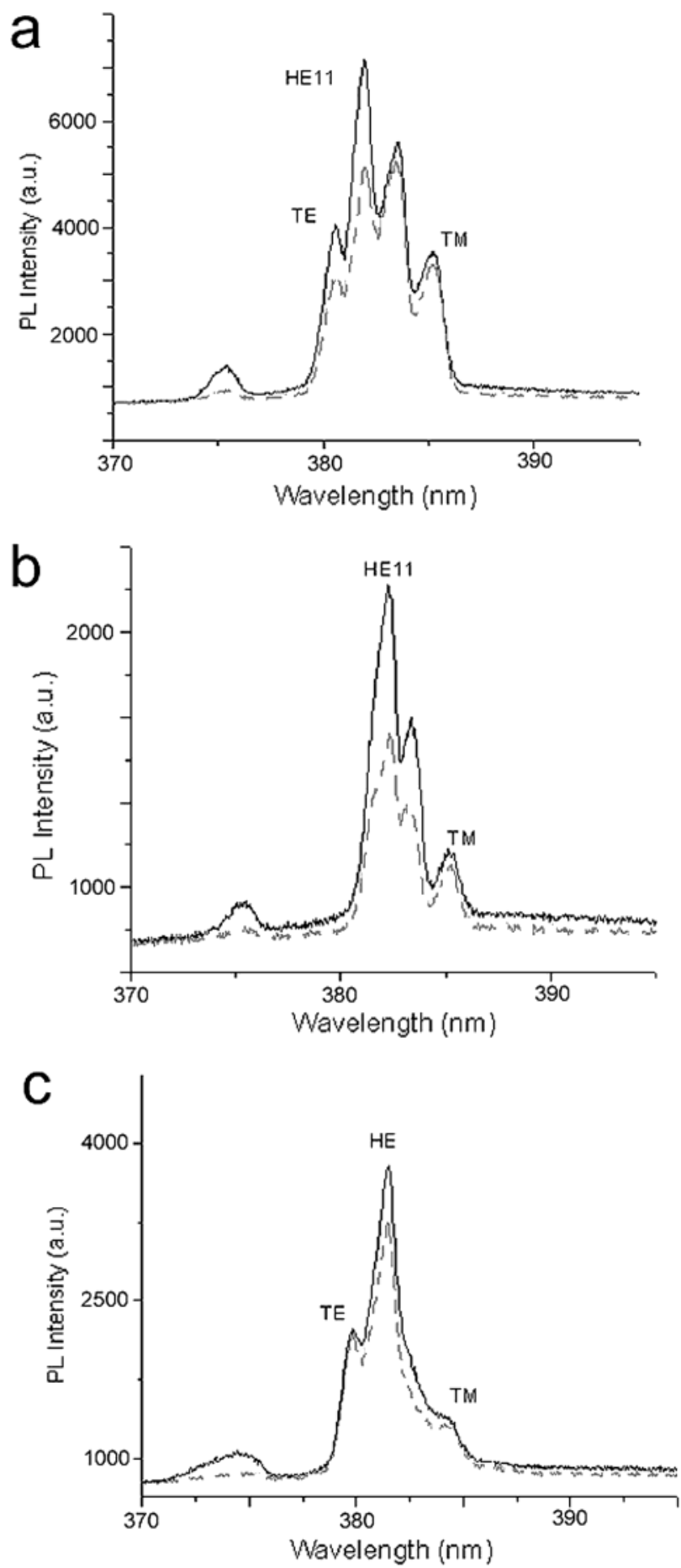

Figure 9. Polarization of individual modes as a function of excitation polarization. (a) Spectra collected for nanowire oriented perpendicular to the incident plane, and incident beam polarized in the plane of incidence. (b) Spectra collected for nanowire oriented perpendicular to the incident plane, and incident polarization parallel to the substrate plane. (c) Spectra collected for incident polarization parallel to the substrate plane, with the nanowire oriented in the plane of incidence. In all cases, the solid curve represents the polarizer oriented to pass $x$-polarized emission.

along the nanowire axis, and maxima at particular values of $\phi$, related to the radius of the nanowire. When the nanowire emission is multimode, the emission pattern will reflect the collective properties of all of the modes. A simulation of this behavior is shown in Figure 10a-c. Experimental examples of the diffracted emission are observed in the images of the cone
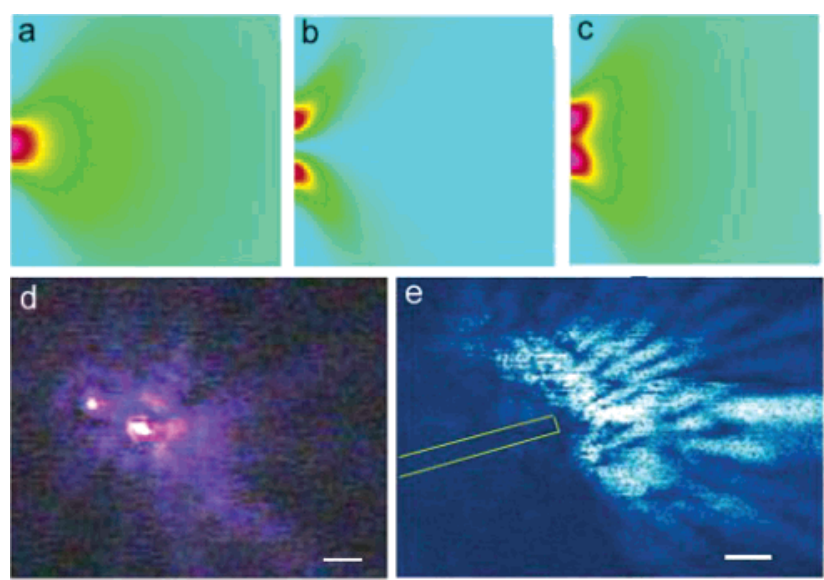

Figure 10. Far-field emission pattern from lasing nanowires. Simulation of the nanowire emission pattern including (a) zeroth order, (b) first order, (c) and sum of zeroth and first order Gaussian electromagnetic field profile. The nanowire is oriented horizontally with the right end face on the left-center edge of the simulation window. (d) Farfield emission pattern of a nanolaser showing multiple angular maxima. Scale bar $=3 \mu \mathrm{m}$. (e) Near-field emission pattern showing additional beam structure. Scale bar $=500 \mathrm{~nm}$. The nanowire position, determined by NSOM topography, is indicated by the yellow line.

emission (Figure 10d). Additional interference effects further modulate the emitted field, is the case for the intricate emission pattern detected in Figure 10e.

C. Pump Power Dependence and Threshold Behavior. The transition from spontaneous PL to a stimulated gain is achieved only upon excitation of a high density of carriers in the nanowire, provided by pulsed UV excitation. The presence of lasing versus spontaneous emission is confirmed by the appearance of narrow cavity modes. These modes have a line width that is in some cases about 1/100 that of the spontaneous emission, and the mode spacing is consistent with cavity dimensions. Figure 11a shows an example of the sharp transition from spontaneous to stimulated emission for a single nanolaser. In Figure $11 \mathrm{~b}$ the spontaneous emission peak narrows smoothly with pump fluence to about $0.55 \Delta v_{0}$. This behavior is probably characteristic of amplified spontaneous emission (ASE), as has been observed in microdisk lasers. ${ }^{39}$ Theoretically, the line width of ASE is given by ${ }^{41}$

$$
\Delta v_{\mathrm{ASE}}=\Delta v_{0}[(G-1) /(G \ln G)]^{1 / 2}
$$

which is 0.62 for typical gain $(G)$ values near $2 \times I_{\text {th }}$. The spatial coherence properties of the beam may also serve to identify it as stimulated or spontaneous, although a direct measure of the spatial coherence has not yet been obtained.

For thinner and shorter nanowires with small mode volume, the transition from spontaneous to stimulated emission is expected to be less distinct, due to a high degree of coupling of spontaneous emission into a single, broad cavity mode. This is the strong-coupling regime, and the criterion for approaching this regime is that $\Delta v_{\text {spont }} / \Delta v_{\mathrm{c}} \leq 1$. This requires a nanowire with $d<130 \mathrm{~nm}$ and $L<2 \mu \mathrm{m}$. We have not yet observed clear lasing behavior in such nanowires, which may not be surprising considering that

$$
g_{\text {th }}=(2 L)^{-1} \ln \left(R^{2}\right)^{-1}
$$

for Fabry-Perot resonators, ${ }^{42}$ resulting in a very large requisite gain $\left(>10^{4} \mathrm{~cm}^{-1}\right)$ to overcome end mirror losses $(R$ is end facet reflectivity). However, some data for wires with length $\sim 4$ $\mu \mathrm{m}$, diameter $\sim 120 \mathrm{~nm}$, (Figure 11c, inset) suggest that the 
a

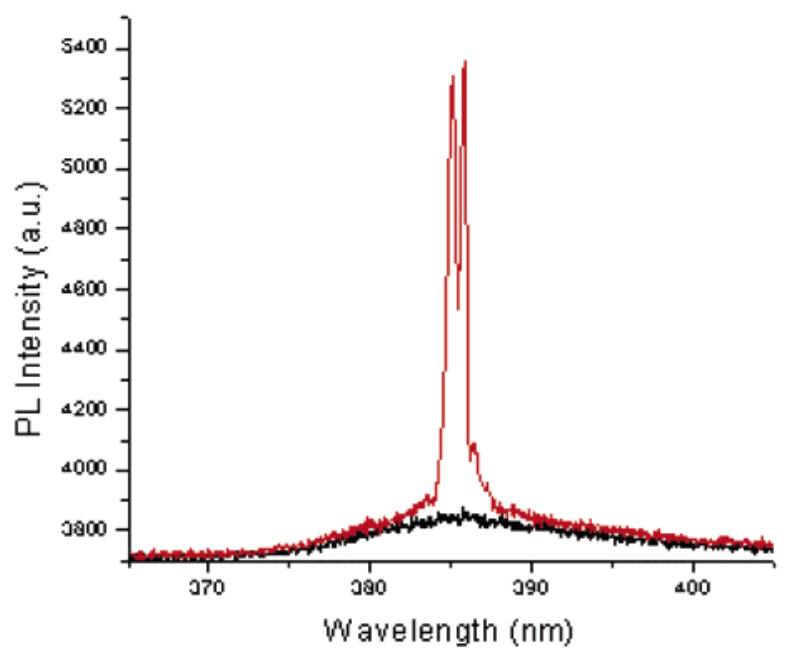

b

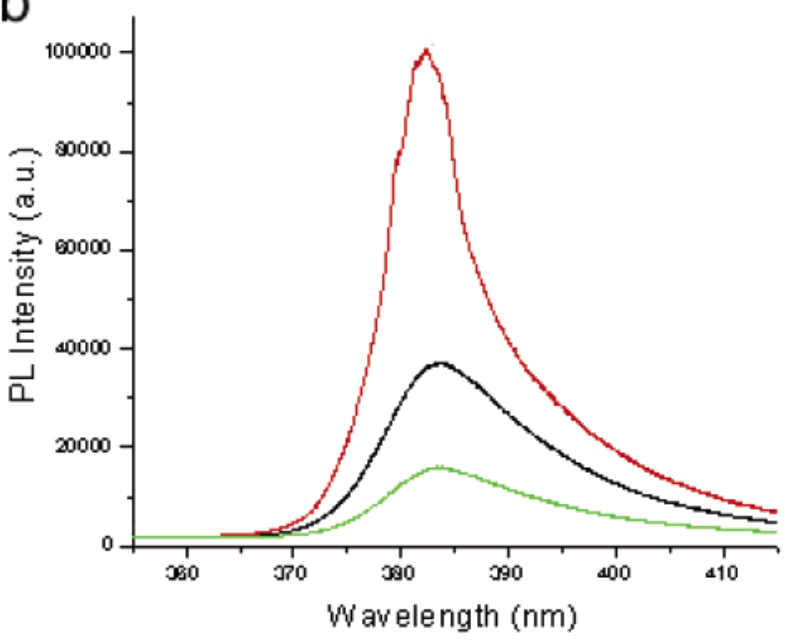

C

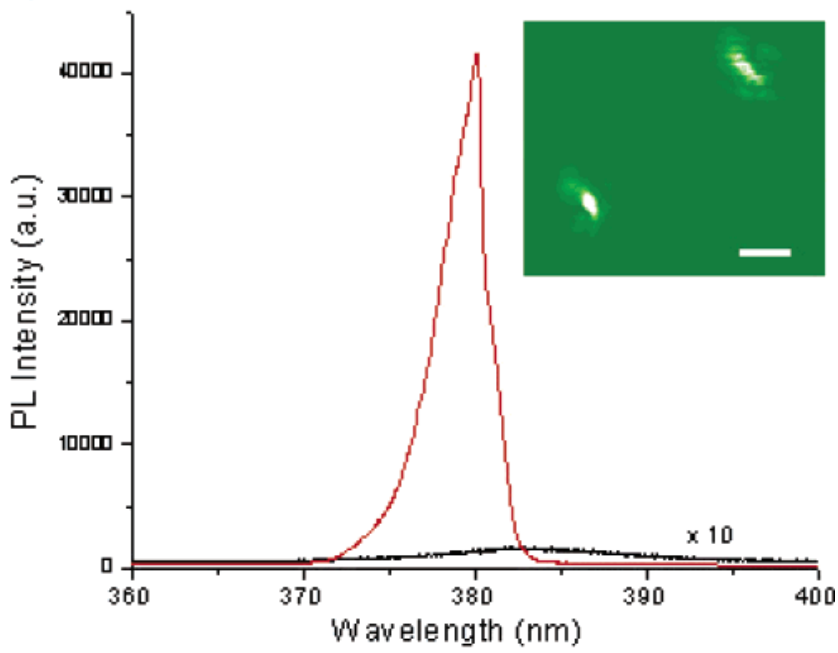

Figure 11. Amplified spontaneous emission vs lasing. (a) Spectra taken at $300 \mathrm{~nJ} / \mathrm{cm}^{2}$ and $450 \mathrm{~nJ} / \mathrm{cm}^{2}$, showing the sharp transition from broad spontaneous emission to lasing. (b) Spectra acquired for $200 \mathrm{~nJ} / \mathrm{cm}^{2}$, $350 \mathrm{~nJ} / \mathrm{cm}^{2}$, and $2000 \mathrm{~nJ} / \mathrm{cm}^{2}$, showing gain narrowing characteristic of ASE in some larger wires. (c) Lasing exhibited in a wire that is 4 $\mu \mathrm{m}$ long. The transition from spontaneous to stimulated emission is less clear in this case. Scale bar $=1 \mu \mathrm{m}$.

strong coupling regime is being approached (Figure 11c). For these wires, spontaneous emission is quite weak, and the first detectable emission exhibits superlinear gain and narrowed line width. The threshold for lasing is also expected to decrease in this regime due to cavity quantum electrodynamics (CQED) ${ }^{16-18}$ although the results obtained so far do not clearly evidence this trend. Elimination of other variables, such as diameterdependence and end face quality, would be crucial for observing this effect.

The lasing thresholds observed in the nanowires vary by orders of magnitude, most likely due to the dimensions, condition of the particular nanowire cavity, and the extent of substrate coupling. The lowest threshold observed for $\mathrm{ZnO}$ nanowires is approximately $70 \mathrm{~nJ} / \mathrm{cm}^{2}$, which is nearly an order of magnitude lower than the lowest observed for $\mathrm{GaN}$ nanowires $\left(\sim 500 \mathrm{~nJ} / \mathrm{cm}^{2}\right) .{ }^{12}$ Pump fluence is used as a measure of the excitation strength as opposed to peak intensity, since the $<200$ fs pulse width is much shorter than PL decay time, thus the influence of the exact pulse duration on the threshold is expected to be minimal. The thresholds observed for the single $\mathrm{ZnO}$ nanowires lead to theoretical threshold gain coefficients on the order of $1000-3000 \mathrm{~cm}^{-1}$ (eq 9). These gain values are somewhat higher than reported for thin films or bulk materials $\left(\sim 180-2000 \mathrm{~cm}^{-1}\right)$, which may be due to higher PL confinement in the nanoscale structures. ${ }^{43-45}$

The power dependences plotted in Figure 12 reveal the highly nonlinear gain and threshold nature of the emission from the nanowires. The spectra in Figure $12 \mathrm{c}$ are taken at various powers, given by the arrows in Figure $12 \mathrm{~b}$. The carrier densities typically excited near threshold and near saturation are estimated from the pump fluence to be approximately $0.2 \times 10^{18} \mathrm{~cm}^{-3}$ and $5 \times 10^{18} \mathrm{~cm}^{-3}$, respectively. Here we assume that all pump photons incident on the nanowire are absorbed, which is reasonable considering the large absorption coefficient $\left(>10^{5}\right.$ $\mathrm{cm}^{-1}$ ) for $\mathrm{ZnO}$ at the pump wavelengths, leading to a short penetration depth $(\sim 100 \mathrm{~nm})$. The Mott density for $\mathrm{ZnO}$ is near $4 \times 10^{18} \mathrm{~cm}^{-3}$, (from above) which theoretically is attained only near saturation $\left(\sim 3-5 \mu \mathrm{J} / \mathrm{cm}^{2}\right)$. Below the critical pump fluence $\left(I_{\mathrm{th}}\right)$, the photoluminescence increases linearly with excitation power, as is expected for free exciton recombination. Once the threshold is reached, a kink in the power dependence is observed, resulting in a large increase in gain. The functional relationship of the power dependence above threshold is in some cases nearly quadratic (as exhibited in Figure 12a), although in other cases it shows linear behavior. At higher pump fluences, typically $2-10 \times I_{\text {th }}$ or at approximately $2-5 \mu \mathrm{J} / \mathrm{cm}^{2}$, nearly complete saturation of the gain is observed. From the theory for a four-level laser, it is expected that pulsed excitation will lead to a gain saturation described as $g=g_{0} \exp \left[-\Gamma / \Gamma_{\mathrm{s}}\right]$, where $g_{0}$ is the gain coefficient at low excitation, $\Gamma$ is the pump fluence, and $\Gamma_{\mathrm{s}}$ is the saturation fluence, given by $h v / \sigma$, where $\sigma$ is the absorption cross-section. ${ }^{41}$ From the value of $\sigma\left(4 \times 10^{-18} \mathrm{~cm}^{2}\right)$ and the photon energy $(4.1 \mathrm{eV})$, the value of $\Gamma_{\mathrm{s}}$ is approximately $0.1 \mathrm{~J} / \mathrm{cm}^{2}$, several orders of magnitude higher than observed here. Clearly, simple gain saturation analysis does not explain the phenomenon observed at low pump fluences here, and thus the transition to from exciton-exciton to EHP dominated gain (discussed below) must cause a perturbation to the lasing scheme that results in the observed power dependence. This could be explained by the increase in transient thermal population of the free electrons, resulting in a modification of the population of the participating lasing levels. Thus, the degree of inversion, given by $\left(N_{2}-N_{1}\right)$, is pinned due to an increasing $N_{1}$ with higher temperatures. $\mathrm{ZnO}$ thin film lasing results have not demonstrated this saturation behavior; however, significantly longer excitation pulse widths are used in those cases, which possibly leads to different EHP dynamics than observed here. 

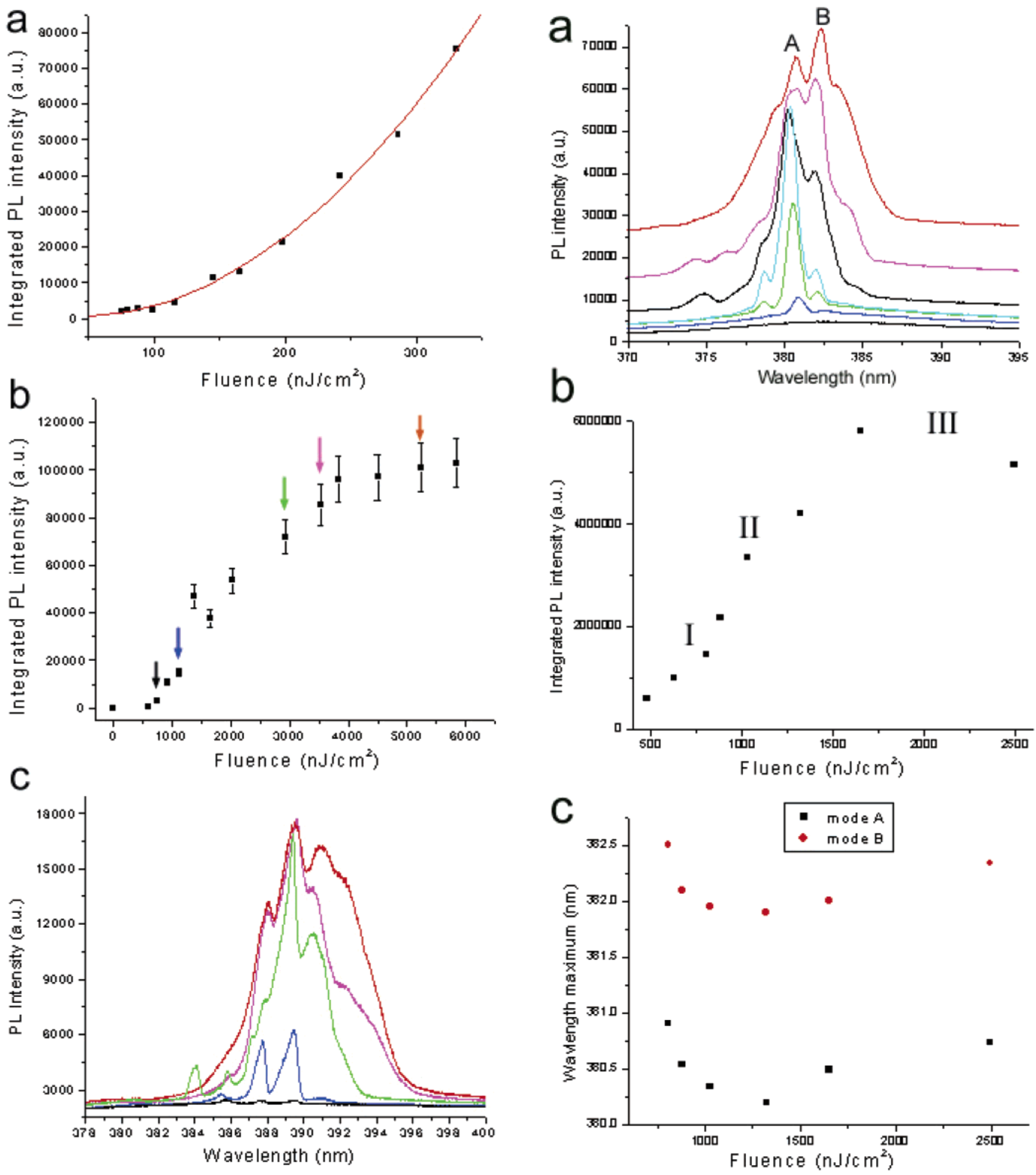

Figure 12. Spectral evolution as a function of incident power. (a) Power dependence for a low threshold laser $\left(80 \mathrm{~nJ} / \mathrm{cm}^{2}\right)$, showing the $I_{\mathrm{p}}{ }^{2}$ dependence of the emission. The red curve is the best-fit to a quadratic function. (b) Overall PL power dependence for a single wire, showing the different excitation regimes. (c) Spectra acquired at the pump fluences given by the colored arrows in (b).

Photoconductivity experiments to determine the precise power dependence of the carrier density, as well as low-temperature experiments, are being performed to further investigate this behavior.

The red-shifts observed in some spectra can be counterbalanced by a blue-shifting caused by an increase in kinetic energy

Figure 13. Shift of individual modes with increasing pump fluence. (a) Spectra, (b) overall power dependence, and (c) shift of individual modes vs pump fluence. The shift of the cavity modes to higher energy suggests a decrease in refractive index due to saturation of exciton absorption.

of the excitons. Band filling weakens exciton binding and nearly exactly cancels the red-shift caused by band gap renormalization (eq 4). This effect is exhibited in the data shown in Figure 13. Figure $13 \mathrm{~b}$ shows an overall power dependence for a single wire, and Figure 13a shows several spectra at different pump fluences $\left(0.5-2.5 \mu \mathrm{J} / \mathrm{cm}^{2}\right)$. The gain profile for the nanolaser is nearly constant at low excitation fluences $\left(<1.2 \mu \mathrm{J} / \mathrm{cm}^{2}\right)$, as 

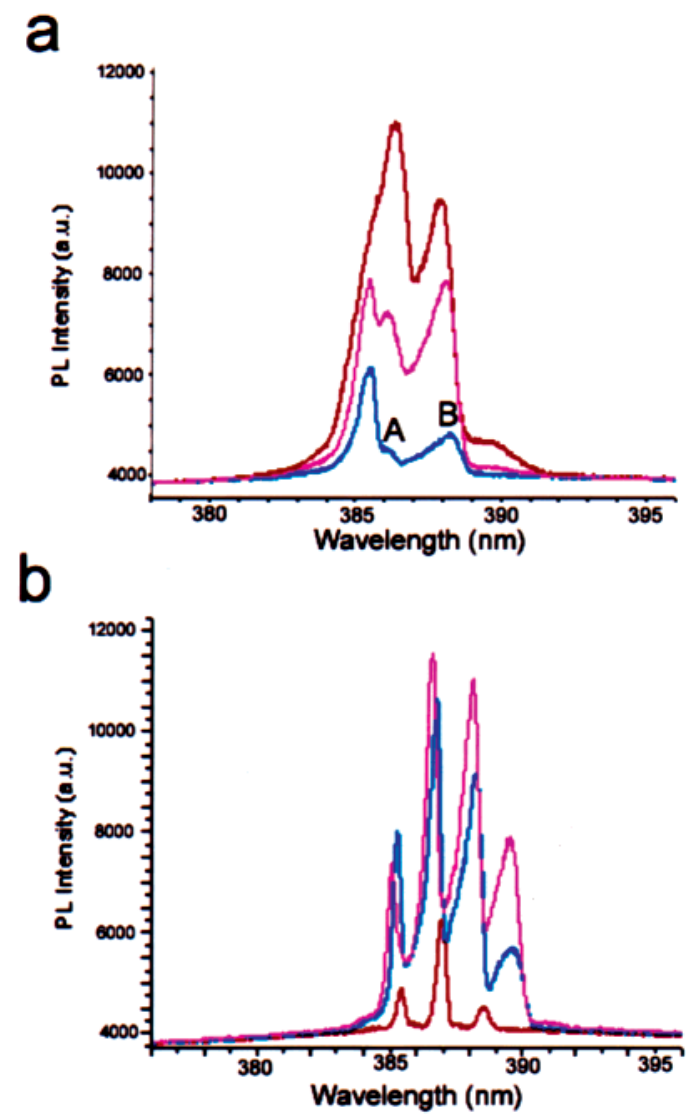

Figure 14. Cavity mode gain as a function of pump fluence. (a) Spectra acquired between $200 \mathrm{~nJ} / \mathrm{cm}^{2}$ and $250 \mathrm{~nJ} / \mathrm{cm}^{2}$ for a $6 \mu \mathrm{m} \mathrm{ZnO}$ wire on sapphire, showing the competition for gain between several progressions of longitudinal cavity modes. (b) Shift of the gain to modes with longer wavelength as a function of pump fluence (red: $425 \mathrm{~nJ} / \mathrm{cm}^{2}$; blue: 640 $\mathrm{nJ} / \mathrm{cm}^{2}$; magenta: $1280, \mathrm{~nJ} / \mathrm{cm}^{2}$ ).

is observed for most $\mathrm{ZnO}$ nanowire lasers. The power dependence is highly nonlinear in this regime (I), which suggests the dominance of excitonic effects. As the fluence increases, the spectrum shifts to lower energy, indicating the decay of excitonic effects $\left(E_{\mathrm{ex}} \rightarrow 0\right)$ and the transition from exciton to EHP dynamics. The kink in the power dependence (II) is indicative of the change in the nature of the dominant carrier, since the EHP is less efficient for radiative recombination. Typically, a roughly linear red-shift of $30-70 \mathrm{meV}$ is observed as the pump fluence exceeds $1-2 \mu \mathrm{J} / \mathrm{cm}^{2}$. The magnitude of the shift is nearly equivalent to that observed in thin films over a similar carrier density range, attributed to exchange energy effects (BGR). ${ }^{46}$

In addition to the shift of the gain curve, a blue-shift of the individual cavity modes is shown in Figure 13c. Shifts of up to $1 \mathrm{~nm}$ were observed with the pump fluences utilized here. This $\Delta \lambda$ correlates with a $0.3 \%$ decrease in the refractive index, given by $\Delta \lambda=2 L \Delta n / m$, where $m$ is the longitudinal mode order. This decrease is reasonable considering the saturation of exciton absorption that has also been observed to accompany the formation of the EHP in CdS. ${ }^{28} \mathrm{~A}$ change in the refractive index also decreases the mode spacing $\propto 1 / n$, however this small change is not measurable given the spectral resolution of the experiment. The $\Delta n$ exhibits a maximum value near the fluence that correlates with the onset of the EHP, then it decreases to near zero as the pump fluence increases. Higher lattice temperatures are likely the cause of the red-shift, since the refractive index of $\mathrm{ZnO}$ increases with temperature. The $\Delta \lambda$ observed suggests a rise in the lattice temperature of ca. $400 \mathrm{~K}$, which is rather large, but reasonable considering the strong coupling of carriers to lattice vibrations in the highly ionic $\mathrm{ZnO}$. Our previous results on $\mathrm{GaN}$ nanowire lasing revealed a consistent red-shift of the gain profile from threshold to saturation and a weak red-shift of the individual modes. In contrast to $\mathrm{ZnO}$, these results suggest that the EHP is dominant in the GaN wires, even near threshold, and that the coupling of the carriers to the lattice temperature is weaker. ${ }^{12}$

The modes observed in the lasing spectrum as a function of power are indicative of the $Q$-factors and gain associated with those modes. Figure 14 shows laser modes as a function of power. In this case, mode B increases in intensity faster than the mode A, despite the possible red-shifting of the gain profile, which indicates that the transverse EM field profile receives a higher net gain. The reason for this is probably a higher degree of confinement of mode B, which would naturally lead to lower loss and larger EM field overlap with the gain medium. Also, the modes in Figure 14a show a distribution of line widths, indicating that they have distinct values of $Q$. The shifting of the gain profile makes it difficult to determine the gain properties of a single lasing mode over a large range of pump fluence, since the mode intensity is a convolution of the material gain profile and cavity properties. The spectra in Figure 14b show several modes as a function of pump power. The modes on the lower energy side of the spectrum receive more gain at higher pump fluence while the modes on the higher energy side receive gain at lower pump fluence and decrease in intensity at the higher pump fluences.

As mentioned previously, the line width of typical nanowire laser emission is approximately $0.25-1.0 \mathrm{~nm}$. A line width of $0.25 \mathrm{~nm}$ (displayed in Figure 15a) corresponds to a value of $Q$ near $1.5 \times 10^{3}$. Given the approximate reflectivity of the end faces $\left(R_{1}=R_{2}=0.19\right)$, the nanowire length $(10 \mu \mathrm{m})$, and no internal losses $T_{\mathrm{i}}=0$, the Fabry-Perot resonator would be expected to have a line width of ${ }^{41}$

$$
\Delta v=-c /(4 \pi L n) \ln \left[R_{1} R_{2}\left(1-T_{\mathrm{i}}\right)^{2}\right]
$$

which is of the order $1.0 \mathrm{~nm}$, in reasonable agreement with the experimental results. The broader line widths of some laser modes indicate some level of internal loss, e.g., diffraction, along the nanowire cavity.

For conventional semiconductor lasers, the line width varies inversely with pump power, due to an increasing degree of population inversion. However, microlasers pumped with short, intense pulses can exhibit additional nonequilibrium effects that act to broaden the lasing peak. ${ }^{47}$ One such effect is shown in Figure $15 \mathrm{~b}$. The laser peak is broadened from 0.5 to $1.3 \mathrm{~nm}$ over the power range of $1.1 \times I_{\text {th }}$ to $1.4 \times I_{\text {th }}$. The broadening occurs primarily on the higher energy side of the mode. It is likely that there is increased spontaneous emission noise at higher pump intensities (due to ASE) that causes phase fluctuations that couple to the real and imaginary parts of the material susceptibility. These optical density fluctuations would couple to the cavity modes and act to broaden the lines, especially in regions with the highest dispersion and absorption. In Figure $15 \mathrm{c}$ it is also observed that the modes near the highest point of the gain spectrum have a narrower line width than those at the edges (especially on the lower energy side). Presumably this results from larger absorption (higher loss) and/or lower gain at the extreme points, leading to a shorter cavity confinement time. Increases of $20 \%$ in the cavity confinement time are calculated from the central mode to the modes near $390 \mathrm{~nm}$ (eq 10). 


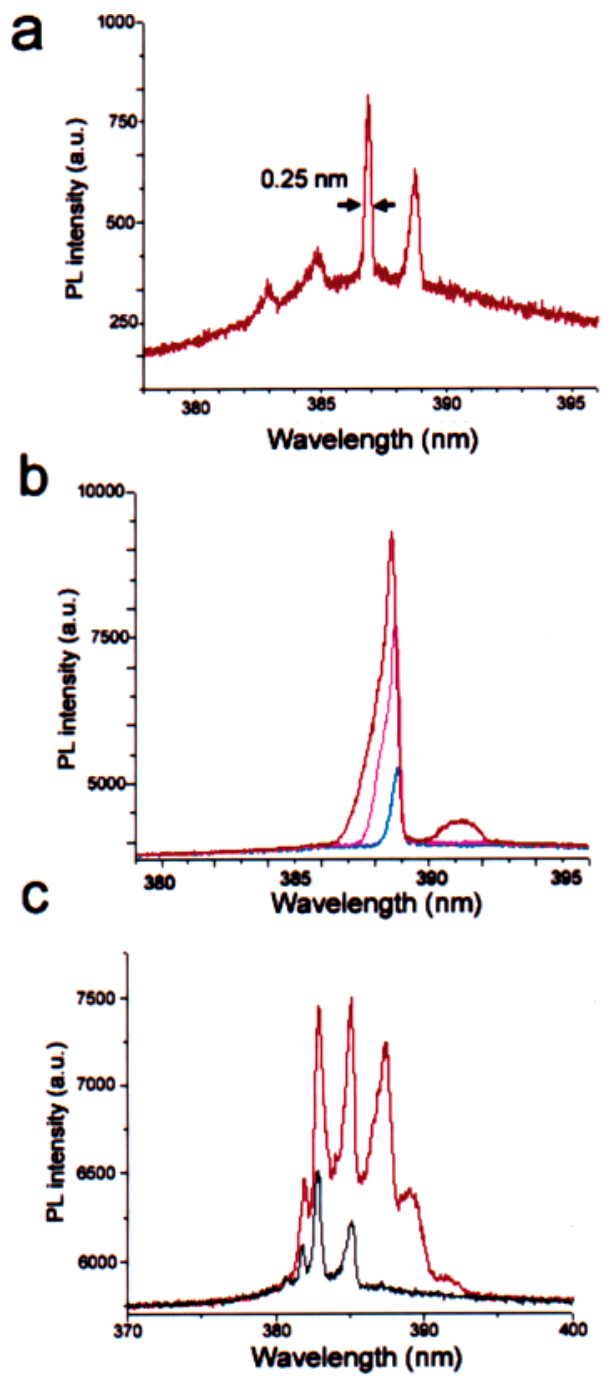

Figure 15. Nanolaser emission line width as a function of pump fluence. (a) Typical narrow laser line width $(0.25 \mathrm{~nm})$. (b) Spectra acquired for pump fluences $180 \mathrm{~nJ} / \mathrm{cm}^{2}$ (blue), $320 \mathrm{~nJ} / \mathrm{cm}^{2}$ (magenta), $760 \mathrm{~nJ} / \mathrm{cm}^{2}$ (red), showing the broadening of the laser peak. A slight blue-shift of the peak of the mode is also apparent. (c) Spectra acquired at $300 \mathrm{~nJ} / \mathrm{cm}^{2}$ (black) and $500 \mathrm{~nJ} / \mathrm{cm}^{2}$ (red), showing the broadening of individual modes that occurs at longer wavelengths.

\section{Conclusions}

The polarization of both the spontaneous and stimulated emission has been characterized for a variety of $\mathrm{ZnO}$ nanowires with different sizes. There is a strikingly distinct behavior for the green versus UV PL, which suggests a strong coupling of the exciton emission to waveguide modes, and strong coupling of the defect PL to the emission dipole parallel to the nanowire axis, possibly due to a surface enhancement for the $\mathrm{V}_{\mathrm{o}} \cdot$ emitting species. Transition to the lasing regime facilitates polarization analysis of the nanowire cavity modes, which are found to behave consistently with theory for a mesoscopic dielectric cylindrical waveguide. Pump power dependence of the laser emission exhibits several trends that can be explained using dispersion and the exciton to EHP transition as a model.

Further study of these systems, including ultrafast transient spectroscopy, on free-standing single nanowires with diameter $<150 \mathrm{~nm}$ should lead to great insight about the CQED and carrier confinement that are responsible for potentially interesting optical phenomena. The size scale necessary for reaching quantum confinement of charge carriers is approximately 1.7 $\mathrm{nm}$, the average Bohr radius for excitons in $\mathrm{ZnO}$. This value has not yet been attained in the synthesis of single- $\mathrm{ZnO}$ crystalline wires; however, other materials such as GaN have a considerable larger exciton radius $(11 \mathrm{~nm})$, making carrier confinement in single wires more practical for these systems. Simultaneous carrier and photonic confinement effects in quasi1D GaN/AlGaN core/shell heterostructures with core size approaching $10 \mathrm{~nm}$ are currently being investigated. ${ }^{48}$

There are many potential applications of the nanowire lasers, and the $\mathrm{ZnO}$ wires studied here have favorable properties. These include high damage threshold, thermal and environmental stability, capability of fabrication of a variety of structures for modification of spatial and spectral emission properties, ${ }^{49,50}$ and potential for electron injection. We expect that future studies should lead to exciting advances in both fundamental nanowire physics and nanoscale optoelectronic and photonic devices.

Acknowledgment. R.J.S. and J.C.J are supported by the Experimental Physical Chemistry Division of the National Science Foundation. P.Y. and H.Y. are supported by the Camille and Henry Dreyfus Foundation, Beckman Foundation, National Science Foundation, and Department of Energy. P.Y. is an Alfred P. Sloan Research Fellow. Work at the Lawrence Berkeley National Laboratory was supported by the Office of Science, Basic Energy Sciences, Division of Materials Science of the U.S. Department of Energy. We thank the National Center of Electron Microscopy for the use of their facilities.

\section{References and Notes}

(1) Bawendi, M. G. Solid State Commun. 1998, 107, 709.

(2) Gérard, J. M.; Sermage, B.; Gayral, B.; Legrand, B.; Costard, E.; Thierry-Mieg, V. Phys. Rev. Lett. 1998, 81, 1110.

(3) Someya, T.; Werner, R.; Forchel, A.; Catalano, M.; Cingolani, R.; Arakawa, Y. Science 1999, 285, 1905.

(4) Wang, J.; Gudiksen, M. S.; Duan, X.; Cui, Y.; Lieber, C. M. Science 2001, 293, 1455.

(5) Cui, Y.; Lieber, C. M. Science 2001, 291, 851.

(6) Kind, H.; Yan, H.; Law, M.; Messer, B.; Yang, P. Adv. Mater. 2002, 14, 158 .

(7) Law, M.; Kind, H.; Kim, F.; Messer, B.; Yang, P. Angew. Chem. 2002, 41, 2405.

(8) Zhang, J.; Chu, D. U.; Wu, S. L.; Ho, S. T.; Bi, W. G.; Tu, C. W.; Tiberio, R. C. Phys. Rev. Lett. 1995, 775, 2678.

(9) Duan, X.; Lieber, C. M. Nature 2003, 421, 241

(10) Huang, M. H.; Mao, S.; Feick, H.; Yan, H.; Wu, Y.; Kind, H.; Weber, E.; Russo, R.; Yang, P. Science 2001, 292, 1897.

(11) Johnson, J. C.; Yan, H.; Schaller, R. D.; Haber, L. H.; Saykally, R. J.; Yang, P. J. Phys. Chem. B 2001, 105, 11387.

(12) Johnson, J. C.; Choi, H.; Knutsen, K. P.; Yang, P.; Saykally, R. J. Nat. Mater. 2002, 1, 106.

(13) Johnson, J. C.; Yan, H.; Schaller, R. D.; Petersen, P. B.; Yang, P.; Saykally, R. J. Nano Lett. 2002, 2, 279.

(14) Wu, Y.; Yang, P. J. Am. Chem. Soc. 2001, 123, 3165.

(15) Snyder, A. W.; Love, D. Optical Waveguide Theory; Kluwer: Boston, 1983.

(16) Ho, S.-T.; Chu, D. Y.; Zhang, J.-P.; Wu, S.; Chin, M.-K. In Optical Processes in Microcavities; Chang, R., Campillo, A., Eds.; World Scientific: Singapore, 1996; pp 339-387.

(17) Ching, E.; Leung, P.; Young, K. In Optical Processes in Microcavities; Chang, R., Campillo, A., Eds.; World Scientific: Singapore, 1996; pp $1-75$.

(18) Brorson, S. D.; Skovgaard, P. M. In Optical Processes in Microcavities; Chang, R., Campillo, A., Eds.; World Scientific: Singapore, 1996; pp 77-97.

(19) Bagnall, D. M.; Chen, Y. F.; Zhu, Z.; Yao, T.; Koyama, S.; Shen, M. Y.; Goto, T. Appl. Phys. Lett. 1997, 70, 2230.

(20) Cao, H.; Zhao, Y. G.; Ong, H. C.; Ho, S. T.; Dai, J. Y.; Wu, J. Y.; Chang, R. P. H. Appl. Phys. Lett. 1998, 73, 3656.

(21) Bagnall, D. M.; Chen, Y. F.; Shen, M. Y.; Zhu, Z.; Goto, T.; Yao, T. J. Crys. Growth 1998, 185, 605-609.

(22) Sun, Y.; Ketterson, J. B.; Wong, G. K. L. Appl. Phys. Lett. 2000, 77, 2322.

(23) Klingshirn, C. Optical Properties of Semiconductors; Springer: New York, 1995; p 306. 
(24) Rubio, J.; Pfeiffer, L.; Szymanska, M. H.; Pinczuk, A.; He, S.; Baranger, H. U.; Littlewood, P. B.; West, K. W.; Dennis, B. S. Solid State Commun. 2001, 120, 423.

(25) Bidnyk, S.; Schmidt, T. J.; Little, B. D.; Song, J. J. Appl. Phys. Lett. 1999, 74, 1-3.

(26) Ambigapathy, R.; Bar-Joseph, I.; Oberli, D. Y.; Haacke, S.; Brasil, M. J.; Reinhardt, F.; Kapon, E.; Deveaud, B. Phys. Rev. Lett. 1997, 78, 3579.

(27) Edjer, E. Phys. Status Solidi 1971, 6, 445.

(28) Kreissl, A.; Bohert, K.; Lyssenko, V. G.; Klingshirn, C. Phys. Status Solidi B 1982, 114, 537.

(29) Sun, X. W.; Kwok, H. S. J. Appl. Phys. 1999, 86, 408.

(30) Sundaram, S. K.; Mazur, E. Nat. Mater. 2002, 1, 217.

(31) Huang, M. H.; Wu, Y.; Feick, H.; Weber, E.; Yang, P. Adv. Mater. 2001, 13, 113.

(32) Yang, P.; Yan, H.; Mao, S.; Russo, R.; Johnson, J.; Saykally, R. J.; Morris, N.; Pham, J.; He, R.; Choi, H.-J. Adv. Func. Mater. 2002, 12, 323.

(33) Schaller, R. D.; Johnson, J. C.; Wilson, K. R.; Lee, L. F.; Haber, L.; Saykally, R. J. J. Phys. Chem. B 2002, 106, 5143.

(34) Egelhaaf, H. J.; Oelgkrug, D. J. Cryst. Growth 1996, 161, 191

(35) Studenikin, S. A.; Covievera, M. J. Appl. Phys. 2002, 91, 5060.

(36) VanHeusen, K.; Warren, W. L.; Seager, C. H.; Tallant, D. R.; Voigt, J. A.; Gnade, B. E. J. Appl. Phys. 1996, 79, 7983.

(37) Landau, L. D.; Lifshitz, E. M.; Pitaevskii, L. P. Electrodynamics of Continuous Media; Pergamon: Oxford, 1984; pp 34-42.
(38) Wang, J.; Gudiksen, M. S.; Duan, X.; Cui, Y.; Lieber, C. M. Science 2001, 293, 1455

(39) Chang, S.; Rex, N. B.; Chang, R. K.; Chong, G.; Guido, L. J. Appl. Phys. Lett. 1999, 75, 166.

(40) Guenther, R. D. Modern Optics; Wiley: New York, 1999; p 341. (41) Svelto, O. Principles of Lasers; Plenum: New York, 1998; p 168.

(42) Domen, K.; Kondo, K.; Kuramata, A.; Tanahashi, T. Appl. Phys. Lett. 1996, 69, 94-96.

(43) Tang, Z. K.; Wong, G. K. L.; Yu, P.; Kawasaki, M.; Ohtomo, A.; Koinuma, H.; Segawa, Y. Appl. Phys. Lett. 1998, 72, 3270.

(44) Chen, Y.; Tuan, N. T.; Sagawa, Y.; Ko, H.-J.; Hong, S.-K.; Yao, T. Appl. Phys. Lett. 2001, 78, 1469.

(45) Yamamoto, A.; Kido, T.; Goto, T.; Chen, Y.; Yao, T.; Kasuya, A. Appl. Phys. Lett. 1999, 75, 469.

(46) Yamamoto, A.; Kido, T.; Goto, T.; Chen, Y.; Takafumi, Y. Solid State Commun. 2002, 122, 29.

(47) Slusher, R. E.; Mohideen, U. In Optical Processes in Microcavities; Chang, R., Campillo, A., Eds.; World Scientific: Singapore, 1996; pp 315337.

(48) Choi, H. J.; Johnson, J. C.; He, R.; Lee, S.; Kim, F.; Pauzauskie, P.; Goldberger, J.; Saykally, R. J.; Yang, P. J. Phys. Chem. B, in press.

(49) Yan, H.; Johnson, J. C.; Law, M.; Yang, P. Submitted.

(50) Yan, H.; He, R.; Johnson, J. C.; Yang, P. J. Am. Chem. Soc. 2003, 125,4728 